\title{
ESTRATÉGIA, LIDERANÇA E CULTURA COMO DIRECIONADORES DE INOVAÇÃO: UM MODELO PARA MICRO E PEQUENAS EMPRESAS BRASILEIRAS
}

\section{STRATEGY, LEADERSHIP AND CULTURE AS INNOVATION VECTORS: A MODEL FOR SMALL BRAZILIAN COMPANIES}

\author{
Gabriela Almeida Marcon, Mestranda ${ }^{1}$ \\ Maria José Hilel Furlani, Bach. ${ }^{2}$ \\ Gabriela Delcin Pires, Bach. ${ }^{3}$
}

(1)Universidade Federal de Santa Catarina (PPEGC/UFSC),-gabriela@almeidamarcon.com

(2) Universidade Federal de Santa Catarina (PPEGC/UFSC)-mjhfurlani@gmail.com

(3) Instituto Federal de Santa Catarina (IFSC)-gabrieladelcinpires@gmail.com

\begin{abstract}
A manutenção da vantagem competitiva depende, atualmente, de iniciativas inovadoras. O fomento da inovação ocorrerá de forma diversa a depender do contexto em que cada organização está inserida. Na economia, o impacto gerado pelas pequenas e médias empresas brasileiras é de extrema importância para o crescimento de emprego e renda em nosso país. Neste contexto, esta pesquisa propõe-se a responder à seguinte questão: Quais critérios integrariam um modelo de processo de inovação para micro e pequenas empresas direcionado pela estratégia, liderança e cultura da organização? O objetivo geral do presente estudo é propor um modelo teórico de processo de inovação voltado para micro e pequenas empresas brasileiras. Apresenta-se uma caracterização das micro e pequenas empresas brasileiras; realizou-se uma revisão da literatura sobre o processo de inovação nas organizações, as características das organizações inovadoras, bem como da cultura e da liderança orientadas à inovação. $\mathrm{O}$ artigo está dividido em cinco seções. O modelo teórico proposto não pretende esgotar a discussão sobre o tema.
\end{abstract}

Palavras-chave: Estratégia, Inovação, Modelo.

Maintaining competitive advantage depends, currently, of innovative initiatives. Fostering innovation is differently depending on the context in which each organization operates. In economics, the impact generated by small and medium Brazilian companies is extremely important for the growth of employment and income in the country. In this context, this research aims to answer the following question: How to propose an innovation process model for small businesses targeted by strategy, leadership and organizational culture? The overall objective of this study is to propose a theoretical model of the innovation process focused on small Brazilian companies. It presents a characterization of small Brazilian companies. It was carried out a review of the literature on the process of innovation in organizations, the characteristics of innovative organizations regarding leadership, culture and strategy. The paper is divided into five sections. The proposed theoretical model does not exhaust the discussion on the subject.

Keywords: Strategy, Innovation, Model.

\section{Introdução}

A inovação, em um contexto complexo, dinâmico, de alta competitividade, vem sendo um objetivo a ser atingido pelas organizações (TIDD et al., 2008; TEZA et al, 2015). Marcial e Grumbach (2002)

apontam a incerteza como sendo uma das características da sociedade da informação. A Administração Estratégica clássica considera que fatores externos interferem na competitividade das empresas (PORTER, 1980), com destaque para a eficiência mercadológica advinda dos ganhos de escala. Na economia brasileira atual, porém, o 


\section{$16^{\circ}$ ERGODESIGN USIHC CINAHPA}

$16^{\circ}$ Ergodesign - Congresso Internacional de Ergonomia e Usabilidade de Interfaces Humano Tecnológica: Produto, Informações Ambientes Construídos e Transporte

$16^{\circ}$ USIHC - Congresso Internacional de Ergonomia e Usabilidade de Interfaces Humano Computador

CINAHPA | 2017 - Congresso Internacional de Ambientes Hipermídia para Aprendizagem. impacto gerado pelas pequenas e médias empresas é de extrema importância para o crescimento de emprego e renda do país, sendo o capital intelectual e a inovação fatores de competitividade (MAIA, 2012). Para Tidd e Bessant (2009) o processo de inovação tem por principais fatores a geração de novas ideias, a seleção das melhores e sua implementação. O empreendedorismo - que vem amadurecendo como disciplina acadêmica (CARRAHER; PARIDON, 2009) - é um aspecto humano que combina estrutura e paixão, planejamento e visão, aliados a sabedoria ao usálos, a energia para executá-los, a oportunidade e a disposição de assumir riscos. (TIDD et al., 2015). Drucker (1985) coloca a inovação como principal aliada do empreendedor. Seria uma nova disciplina, que pode ser aprendida e praticada. Está relacionada, essencialmente, com aprendizagem e mudança e, em geral, é disruptiva, onerosa e envolve risco (DRUCKER, 1985; TIDD et al., 2015). Sabe-se que as organizações, a fim de transformar seu modo de executar atividades ou mesmo desenvolver algo novo, necessitam adaptar suas estratégias, suas equipes e, invariavelmente, ter ou construir uma cultura voltada à inovação (TIDD et al., 2015). A cultura organizacional tem por alicerce um plexo axiológico compartilhado por toda a equipe e relacionado aos valores que influenciam as pessoas em seu ambiente social (NEVES, 2001; ÇAKAR; ERTÜRK, 2010). Neste contexto, esta pesquisa propõe-se a responder à seguinte questão: Quais critérios integrariam um modelo de processo de inovação para micro e pequenas empresas direcionado pela estratégia, liderança e cultura da organização? O objetivo geral do presente estudo é propor um modelo teórico de processo de inovação voltado para micro e pequenas empresas brasileiras. A aplicação do modelo teórico criado não foi objeto desta pesquisa em particular.

O artigo está dividido em cinco seções: introdução, referencial teórico, enquadramento metodológico, discussão dos resultados e considerações finais.

\section{Referencial Teórico}

A difusão de novas práticas e tecnologias há tempos atrai atenção de pesquisadores e gestores (BASS, 1969; TEZA et. al, 2015;

DESMARCHELIER; FANG, 2016). Os processos de difusão da inovação requerem, principalmente, as características da própria inovação, os canais de comunicação entre os interessados, um sistema social que conecte os agentes, além do fator tempo (ROGERS, 1962). As decisões tomadas no início do processo de inovação tendem a conduzir as demais (AAGAARD; GERTSEN, 2011). Nem todos os componentes do processo de inovação podem ser controlados pelo gestor, fatores e intervenientes externos podem interferir nas ações inovadoras (CARUANA et al., 2002; PRAJOGO, 2016).

As organizações, em geral, não inovam de forma isolada, mas interagindo umas com as outras (FAGERBERG, 2006). Para Tidd et al. (2008) uma organização inovadora sugere mais que uma estrutura; cuida-se de um plexo de componentes que operam em conjunto para criar e fortalecer o tipo de ambiente que propicia a inovação. Em se tratando de inovação em serviços, duas abordagens são fundamentais, a saber: inovação do processo de prestação do serviço e inovação em termos de novas ofertas que satisfaçam os desejos e necessidades dos clientes (BERRY et. al, 2006), atividades que dependem do compartilhamento de conhecimentos entre os membros da equipe (HUSSAIN et. al, 2016).

As motivações para inovar podem ser econômicas ou sociais, razões que podem, inclusive, estar interligadas, reforçando-se mutuamente (KENNEDY; FISS, 2009). A cultura organizacional também é um aspecto importante para o fomento da inovação (SCHEIN, 2004; YANG, 2007; LOVE; CEBON, 2008; BINA, 2012; SZCZEPAŃSKA-WOSZCZYNA, 2015; HUSSAIN et. al, 2016). A base da cultura organizacional, é o aprendizado em conjunto de crenças, valores e pressupostos (SHEIN, 1999; SCHEIN, 2004; MORCILLO, 1997). Há evidências de que empresas de pequeno porte estão restritas a oportunidades cujas perspectivas de expansão continuada são limitadas (PENROSE, 1959). Assim, não é o valor gasto em pesquisa e desenvolvimento que determina o quão inovadora uma empresa é, mas a maneira como aloca os recursos disponíveis (LEUZINGER e

FERNANDES, 2012). Tidd e Bessant (2009), portanto, apontam que as organizações, no que diz respeito ao processo de inovação, devem selecionar, estrategicamente, do conjunto de gatilhos potenciais para inovação as coisas com que a empresa comprometerá recursos para execução. O Brasil é conhecido por sua heterogeneidade cultural, oriunda de sua colonização e influência de diversas culturas e 


\section{$16^{\circ}$ ERGODESIGN USIHC CINAHPA}

$16^{\circ}$ Ergodesign - Congresso Internacional de Ergonomia e Usabilidade de Interfaces Humano Tecnológica: Produto, Informações Ambientes Construídos e Transporte

$16^{\circ}$ USIHC - Congresso Internacional de Ergonomia e Usabilidade de Interfaces Humano Computador

CINAHPA | 2017 - Congresso Internacional de Ambientes Hipermídia para Aprendizagem. crenças. Esse fato se reflete na identidade cultural organizacional, igualmente heterogênea, logo, considerar uma unidade cultural traz resultados falaciosos (ALCADIPANI; CRUBELLATE, 2003). As empresas brasileiras costumam se espelhar em culturas organizacionais do exterior

(ALCADIPANI; CRUBELLATE, 2003; CHU; WOOD, 2008), o que tende a ser prejudicial para as práticas nacionais, pois o Brasil possui traços culturais típicos (CALDAS, 1997; CHU; WOOD, 2008). A estratégia deve, portanto, considerar a heterogeneidade das empresas, uma vez que é aprimorada quando se leva em conta as competências específicas e distintivas, as dotações de recursos de cada organização (PENROSE, 1959; WERNERFELT, 1995).

Os processos de inovação requerem alto envolvimento e atividades intensivas em conhecimento (ROSSETI et al., 2008). A partir da década de 1980, passou-se a considerar que a inovação apresenta caráter sistêmico (FAGERBERG, 2006). Para Isaacson (2014), as forças sociais e culturais compõem o ambiente da inovação. Nos últimos tempos, novas formas de colaboração surgiram (TAPSCOTT; WILLIAMS, 2007).

A liderança organizacional, consequentemente, desempenha papel fundamental ao agregar e influenciar o grupo, a fim de catalisar resultados e alcançar objetivos previamente delineados (YUKL, 1994; BARRETO et al., 2013; ZANELI et al., 2014; NORTHOUSE, 2013). A percepção dos usuários é outro aspecto a ser considerado (TIDD et al., 2015), pois alguns usuários estão à frente da indústria no que tange às necessidades de inovação (VON HIPPEL, 2005). Diamond (1997), por sua vez, afirma que as invenções criam as necessidades dos usuários. As inovações podem ser, de modo geral, incrementais ou disruptivas. Serão incrementais aquelas que acrescentarem um diferencial àquilo que já existe, seja agregando valor a um produto, seja prestando o mesmo serviço de forma aprimorada (TIDD et al., 2015). A aprendizagem organizacional é um aspecto fundamental resultante do processo de inovação e, de acordo com Lundvall (1992), pode se manifestar de três distintas formas: aprendizado pela prática (learning by doing); aprendizado pelo uso (learning by using) e aprendizado pela interação dos usuários e produtores (learning by interacting). Em razão da abundância de recursos naturais, o Brasil tem potencial para desenvolver muitas inovações, mas as empresas ainda investem pouco em $\mathrm{P} \& \mathrm{D}$, devendo avançar e estruturar seus processos (LEUZINGER; FERNANDES, 2012). A inovação de processos pode ser entendida como situação em que especialistas criam ideias pertinentes e originais sobre como processos poderiam ser redesenhados (FIGL; RECKER, 2016).

Bons modelos de fluxo podem ser determinantes no sucesso da implementação de melhorias nos processos de inovação empresariais (FIGL e RECKER, 2016). Um modelo é uma representação da realidade, que permite ao gestor ampliar sua visão e explorar cenários futuros (PIDD, 1997), por isso a importância de um que contemple as micro e pequenas empresas.

\section{Método}

A princípio, foi realizada uma pesquisa teórica básica, a partir da revisão sistemática da literatura em base de dados científica. Após, os pesquisadores construíram um modelo teórico de fluxo representativo do processo de inovação para micro e pequenas empresas, considerando a realidade brasileira. Quanto à natureza do objetivo, a pesquisa é exploratória, por fomentar a reflexão acerca do tema. A pesquisa exploratória pretende fazer um levantamento para buscar evidências científicas (TRANFIELD et al, 2003). No que tange à abordagem do problema, a pesquisa classifica-se como qualitativa, por ser um instrumento para exploração e compreensão do significado que os indivíduos ou grupos atribuem a um problema social ou humano (CRESWELL, 2010).

\section{Discussão dos Resultados}

Diante da dificuldade em criar departamentos especializados para investigação intensiva de oportunidades de inovação, os pequenos e microempresários devem focar no conhecimento vasto que podem extrair dos usuários de produtos e serviços, os quais, muitas vezes, antevê em soluções ainda não propostas pelo mercado (VON HIPPEL, 2005). A cultura e a liderança organizacional naturalmente voltadas à inovação podem ser consideradas recursos empresariais distintivos, uma vez que outras micro e pequenas empresas dificilmente conseguirão desenvolver estas competências num curto espaço de tempo (PENROSE, 1959). A inovação em pequenos 


\section{ERGODESIGN USIHC CINAHPA}

negócios tende a ser incremental (TIDD et al., 2015). O modelo teórico a seguir proposto pretende servir de norte para futuras investigações e estudos empíricos acerca do fluxo para os processos de inovação das pequenas empresas brasileiras. A Figura 1 ilustra a fase inicial de implementação da inovação em organizações de pequeno porte:

Figura 1 - Fluxo da Inovação em Micro e Pequenas Empresas - Fase 1 - Identificação das Competências

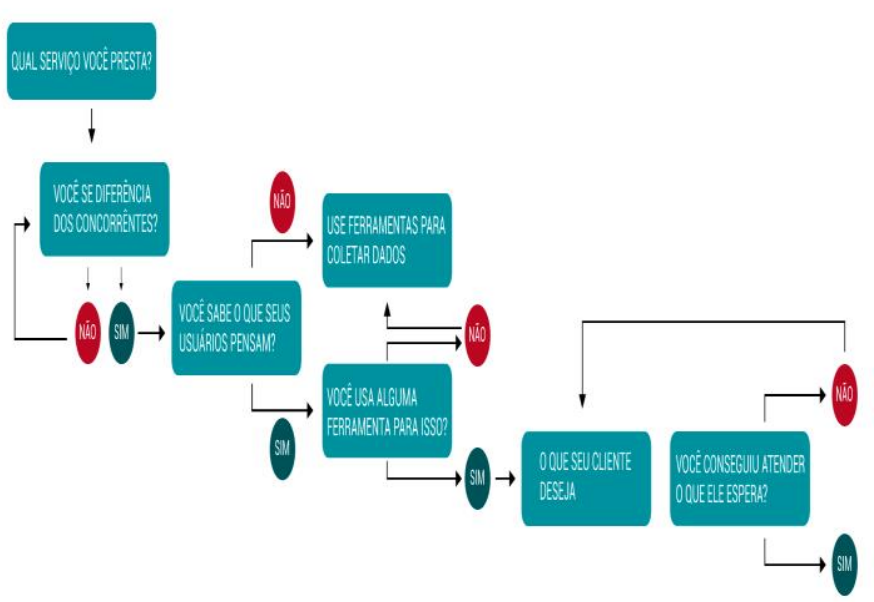

Fonte: Elaborado pelos autores a partir dos dados da pesquisa (2016).

Considerando a importância do usuário para o processo de inovação das micro e pequenas empresas, é necessário que o gestor identifique o público-alvo do negócio e o que este público considera importante, a fim de agregar valor aos produtos e serviços oferecidos a eles.

Após a identificação das competências distintivas da organização e de seu público-alvo, o gestor deve buscar identificar as lacunas no seu segmento e avaliar as oportunidades do mercado, através de pesquisas e/ou interação direta com os usuários, com objetivo de criar ou agregar valor ao que já faz, incrementando seus produtos ou serviços. Só após este diagnóstico das demandas é que a organização passará à fase de prototipação de sua inovação.

Com a caracterização das competências organizacionais, do público-alvo e das oportunidades de inovação, as micro e pequenas empresas passam à fase de implementação das inovações em seus produtos ou serviços. A aprendizagem organizacional, ao final do processo, contribui para que seja constante a melhoria dos processos inovativos organizacionais. $16^{\circ}$ Ergodesign - Congresso Internacional de Ergonomia e Usabilidade de Interfaces Humano Tecnológica: Produto, Informações Ambientes Construídos e Transporte

$16^{\circ}$ USIHC - Congresso Internacional de Ergonomia e Usabilidade de Interfaces Humano Computador

CINAHPA | 2017 - Congresso Internacional de Ambientes Hipermídia para Aprendizagem.
O fluxo completo proposto pode ser verificado na Figura 2:

Figura 2 - Fluxo da Inovação em Micro e Pequenas Empresas
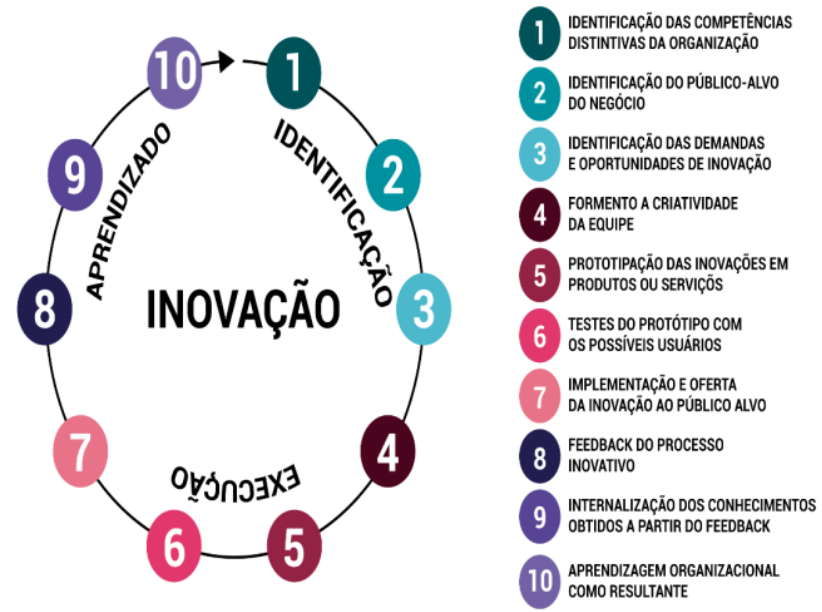

Fonte: Elaborado pelos autores (2016).

Em todas as fases do modelo a atuação da liderança voltada à inovação é fundamental, no sentido de que incentiva e une o grupo em torno dos objetivos organizacionais, viabilizando a profusão de novas ideias e a conjugação de esforços em torno do aprimoramento dos produtos e serviços, bem como incentivo ao contato direto com os usuários. A retroalimentação do fluxo através do feedback dos usuários e da equipe ao final é essencial para que a organização se mantenha em constante evolução. Além disso, a aprovação e testes dessas fases, pode diminuir um erro em uma fase final, pois propõe que haja a validação dessas durante o ciclo.

O fluxograma mostra o processo da inovação como algo cíclico, ou seja, ele deve ser continuo, estar em constante manutenção, e também deve ter as suas fases testadas continuamente, num mecanismo de retroalimentação.

\section{Considerações Finais}

O estudo demonstrou que o processo de inovação nas empresas tem por vetores a estratégia, a liderança e a cultura organizacionais. A Inovação então surgiria de um processo encadeado em que produtos serviços ou processos são melhorados ou criados a fim de que a organização inovadora possa avançar, competir e 


\section{$16^{\circ}$ ERGODESIGN USIHC CINAHPA}

$16^{\circ}$ Ergodesign - Congresso Internacional de Ergonomia e Usabilidade de Interfaces Humano Tecnológica: Produto, Informações Ambientes Construídos e Transporte

$16^{\circ}$ USIHC - Congresso Internacional de Ergonomia e Usabilidade de Interfaces Humano Computador

CINAHPA | 2017 - Congresso Internacional de Ambientes Hipermídia para Aprendizagem. diferenciar-se com sucesso em seu mercado. $\mathrm{O}$ sucesso da inovação parece depender não apenas dos recursos de que dispõe a organização, mas de sua capacidade de geri-los da melhor forma possível.

No Brasil é alto o índice de negócios que nascem da necessidade de subsistência, não de oportunidades criadas para o empreendimento. A mortandade das pequenas empresas ocorre, por vezes, em virtude da falta de conhecimento no ramo do negócio escolhido e por não saber como implementar uma inovação em seus processos, produtos ou serviços.

O modelo apresentado não é necessariamente válido para toda e qualquer realidade, mas serve como facilitador para que pequenas e microempresas possam incrementar inovações com intuito de satisfazer seus clientes e ganhar novos mercados.

\section{Referências Bibliográficas}

AAGAARD, Annabeth; GERTSEN, Frank.

Supporting radical front end innovation: perceived key factors of pharmaceutical innovation.

Creativity and Innovation Management, v. 20, n. 4, p. 330-346, 2011.

ALCADIPANI, Rafael; CRUBELLATE, João

Marcelo. Cultura organizacional: generalizações improváveis e conceituações imprecisas. Rev. adm. empres., São Paulo, v. 43, n. 2, p. 64-77, Junho 2003 .

BASS, Frank M. A new product growth for model consumer durables. Management Science, v. 15, n. 5, p. 215-227, 1969.

BARRETO L. M. T. S., KISHORE A., REIS G. G., BAPTISTA L. L., MEDEIROS C. C. Cultura organizacional e liderança: uma relação possível?

R.Adm., São Paulo, v.48, n.1, p-34-52, 2013. BERRY, L. L., SHANKAR, V., PARISH, J. T., CADWALLADER, S., DOTZEL, T. Creating new markets through service innovation. MIT Sloan

Management Review, 47(2), 56, 2006.

BINA, S. S., Insigniam Corporate

CultureReport2012.Insigniam Performance 2012. Disponível em: <http://insigniam.com/staging/wpcontent/uploads/2012/08/Insigniam_Corp-CultureReport.pdf $>$ Acesso em: 31.08.2016.

CALDAS, M. Santo de casa não faz milagre:

condicionantes nacionais e implicações

organizacionais da fixação brasileira pela figura do "estrangeiro". M. Cultura organizacional e cultura brasileira. São Paulo: Atlas, 1997.
CARRAHER, Shawn M.; PARIDON, Terrence J. Entrepreneurship journal rankings across the discipline. Journal of Small Business Strategy, v. 19, n. 2, p. 89-98, 2009.

CARUANA, Albert; EWING, Michael T.;

RAMASESHAN. Effects of some environmental challenges and centralization on the entrepreneurial orientation and performance of public sector entities. Service Industries Journal, v. 22, n. 2, p. 43-58, 2002.

ÇAKAR, Nigar Demircan; ERTÜRK, Alper. Comparing innovation capability of small and medium-sized enterprises: examining the effects of organizational culture and empowerment. Journal of Small Business Management, v. 48, n. 3, p. 325-359, 2010.

CRESWELL, J. W. Projeto de pesquisa: Métodos qualitativo, quantitativo e misto. 3. ed. Porto Alegre: Artmed, 2010.

DESMARCHELIER, Benoît; FANG, Eddy S.

National culture and innovation diffusion.

Exploratory insights from agent-based modeling.

Technological Forecasting and Social Change, v. 105, p. 121-128, 2016.

DIAMOND, Jerry. Guns, Germs, and Steel: The

Fates of Human Societies. New York: WW Norton \& Company, 1997.

DRUCKER, P.; Innovation and

Entrepreneurship. Harper \& Row, New York, 1985.

FAGERBERG, J. Systems of Innovation: perspectives and challenges. In FAGERBERG, J.; MOWERY, D.C.; Nelson, R. (orgs.) The Oxford

Handbook of Innovation. Oxford University Press, 2006.

FIGL, Kathrin; RECKER, Jan. Process innovation as creative problem solving: An experimental study of textual descriptions and diagrams. Information \& Management, 2016.

GEM - The Global Entrepreneurship

Monitor.2015 Global Report. Disponível em: < http://www.gemconsortium.org/report>. Acesso em: 07.09.2016.

HUSSAIN, Kashif; KONAR, Rupam; ALI, Faizan. Measuring Service Innovation Performance through Team Culture and Knowledge Sharing Behaviour in Hotel Services: A PLS Approach.

Procedia-Social and Behavioral Sciences, v. 224, p. 35-43, 2016.

IPEA - Instituto de Pesquisa Econômica Aplicada.

O Panorama das Políticas Públicas Federais

Brasileiras Voltadas para as Empresas de 


\section{$16^{\circ}$ \\ ERGODESIGN USIHC CINAHPA}

$16^{\circ}$ Ergodesign - Congresso Internacional de Ergonomia e Usabilidade de Interfaces Humano Tecnológica: Produto, Informações Ambientes Construídos e Transporte

$16^{\circ}$ USIHC - Congresso Internacional de Ergonomia e Usabilidade de Interfaces Humano Computador

CINAHPA | 2017 - Congresso Internacional de Ambientes Hipermídia para Aprendizagem.
Pequeno Porte. 2016. Disponível em:

http://www.ipea.gov.br/portal/index.php?option=co $\mathrm{m}$-content\&view $=$ article\&id $=28297 \&$ Itemid $=406$ Acesso: 05.09.2016.

ISAACSON, Walter. Os inovadores: uma biografia da revolução digital. Companhia das letras: São Paulo, 2014.

KENNEDY, Mark Thomas; FISS, Peer Christian. Institutionalization, framing, and diffusion: The logic of TQM adoption and implementation decisions among US hospitals. Academy of Management Journal, v. 52, n. 5, p. 897-918, 2009.

LEUZINGER, Roberto; FERNANDES, Fernando. Como alavancar a inovação no Brasil. Harvard Business Review Brasil, web log post, v. 7, 2012. LOVE, E. Geoffrey; CEBON, Peter. Meanings on Multiple Levels: The Influence of Field-Level and Organizational-Level Meaning Systems on Diffusion. Journal of Management Studies, v. 45, n. 2, p. 239-267, 2008.

LUNDVALL, B. National System of Innovation: Towards a theory of innovation and interactive learning. London-New York, Pinter Publishers: 1992.

MAIA, A. F. da S. Inovação em Micro e

Pequenas Empresas: Uma Análise do Caso

Brasileiro. Dissertação apresentada ao Instituto de Economia da Universidade Federal de Uberlândia. Uberlândia. 2012.

MARCIAL, Elaine Coutinho, GRUMBACH, Raul José dos Santos. Cenários prospectivos: como construir um futuro melhor. Rio de Janeiro: FGV, 2002.

MORCILLO, P. Strategic management of technology and innovation. Madrid: Civitas, 1997. MPEs ativas no Brasil.Disponível em $<$ http://empresometro.cnc.org.br/Estatisticas $>$ acesso em 07.09.2016.

NEVES, José Gonçalves das. Clima

organizacional, cultura organizacional e gestão de recursos humanos. Lisboa: Editora RH, 2001. NORTHOUSE, Peter G., Leadership: theory and practice, 6 ed. SAGE, 2013

PENROSE, Edith. The Theory of the Firm. NY:

John Wiley \& Sons, 1959.

PIDD, Michael. Tools for thinking-Modelling in management science. Journal of the Operational

Research Society, v. 48, n. 11, p. 1150-1150, 1997. PORTER, M. E. Competitive Strategy. New York: Free Press, 1980.

PRAJOGO, Daniel I. The strategic fit between innovation strategies and business environment in delivering business performance. International

Journal of Production Economics, v. 171, p. 241249, 2016.

ROGERS, E.M., Diffusion of Innovations. The Free Press, 1962.

ROSSETTI, A., PACHECO, A. P. R., SALLES, B., GARCIA, M., \& SANTOS, N. A organização baseada no conhecimento: novas estruturas, estratégias e redes de relacionamento. Ciência da Informação, 37(1), 61-72. 2008.

SCHEIN, E. H. The corporate culture- survival guide. Jossey-Bass: São Francisco, 1999.

SCHEIN, E. H. Organizational culture and leadership. 3. ed. Jossey-Bass: São Francisco, 2004

SZCZEPAŃSKA-WOSZCZYNA, Katarzyna.

Leadership and organizational culture as the normative influence of top management on employee's behaviour in the innovation process.

Procedia Economics and Finance, v. 34, p. 396402, 015.

TAPSCOTT, Don; WILLIAMS, Anthony D.

Wikinomics: Como a colaboração em massa pode mudar o seu negócio. Tradução Marcello Lino. Rio de Janeiro: Nova Fronteira, 2007.

TEZA, P., DANDOLIN, G., de SOUZA, J. A., MIGUEZ, V. B., FERNANDES, R. F., \&MIGUEL, P. A. C. Modelos de front end da inovação: similaridades, diferenças e perspectivas de pesquisa. Production, 25(4), 851-863, 2015. TIDD, Joe. BESSANT, John. Inovação e Empreendedorismo: Administração. Bookman Editora, 2009.

TIDD, Joe; BESSANT, John; PAVITT, Keith.

Gestão da Inovação. $3^{\text {a }}$ edição. Artmed Editora, 2008.

TIDD, Joe; BESSANT, John; PAVITT, Keith. Gestão da Inovação. $5^{\text {a }}$ edição. Bookman Editora, 2015.

TRANFIELD, D.; DENYER, D.; SMART, P. Towards a methodology for developing evidenceinformed management knowledge by means of systematic review. British Journal of

Management, v. 14, n. 3, p. 207-222, 2003.

VON HIPPEL, Eric. Democratizing innovation:

The evolving phenomenon of user innovation.

Journal für Betriebswirtschaft, v. 55, n. 1, p. 6378, 2005.

YANG, Jen-Te. Knowledge sharing: Investigating appropriate leadership roles and collaborative culture. Tourism Management, v. 28, n. 2, p. 530- 
$16^{\circ}$ USIHC - Congresso Internacional de Ergonomia e Usabilidade de CINAHPA

Interfaces Humano Computador

CINAHPA | 2017 - Congresso Internacional de Ambientes Hipermídia para Aprendizagem.

543, 2007.

YUKL, G. Leadership in organizations.

Englewood Cliffs: Prentice Hall, 1994.

ZANELLI J. C., ANDRADE J. E. B., BASTOS A.

V. B., Psicologia, Organizações e Trabalho no

Brasil, 2. ed, Artmed, Porto Alegre, 2014. 